\title{
3D printing orthosis with propulsion system increases pelvic rotation in gait of children with cerebral palsy
}

\author{
Mariana Volpini Lana ${ }^{1,2,3^{*}}$, Mariana Aquino ${ }^{2,3}$, Anderson Horta ${ }^{3}$, Paulo Reis $^{3}$, Joana Maia ${ }^{3}$, Sérgio T. \\ Fonseca $^{2}$ \\ ${ }^{1}$ Faculdade de Ciências Médicas de Minas Gerais \\ Alameda Ezequiel Dias, 275 - Centro, Belo Horizonte, MG, Brasil \\ *marianavolpini@amr.org.br \\ ${ }^{2}$ Universidade Federal de Minas Gerais \\ Av. Pres. Antônio Carlos, 6627 - Pampulha, Belo Horizonte, MG, Brasil \\ marircaquino@gmail.com; sergioteixeirafonseca@gmail.com \\ ${ }^{3}$ Associação Mineira de Reabilitação \\ Rua Prof. Otávio Coelho de Magalhães, 111, Mangabeiras, Belo Horizonte, MG, Brasil \\ andersonhorta@gmail.com; paulogsk@gmail.com; joanapimentato@gmail.com
}

\begin{abstract}
INTRODUCTION: Despite the short- and long-term benefits, the continuous use of orthotics causes impairments due to movement restriction, such as disuse atrophy of ankle plantar flexor muscles. Consequently, an inefficient push-off might be present, generating kinematic compensations in distant regions of the ankle (e.g. pelvis), due to force transmission thru interactions of the body kinetic chain. Thus, the objective of present pilot study is to verify whether there is a difference between conventional polypropylene ankle foot orthosis (AFO) and tridimensional (3D) printing AFO with and without ankle external propulsion system, in pelvis kinematics during the gait of children with cerebral palsy (CP). METHODS: This pilot study investigated the plegic lower limb kinematic data, during self-selected walking speed, of five children with hemiplegic CP (5 to 17 years old) were collected with a 3D motion analysis system. One-way ANOVA repeated measures was used to compared pelvis frontal and transverse plane amplitudes in two hinged AFO on three conditions - conventional, 3D printing with and without propulsion system. RESULTS: Pelvis amplitude in transverse plane showed differences between conditions $(\mathrm{p}=0,04)$, indicating that AFO with propulsion system increases pelvic rotation during gait. That is, more energy might reach pelvis coming from the ankle, through $3 \mathrm{D}$ printing AFO with propulsion system, increasing pelvis transverse plane movement, and facilitating the swing of the contralateral limb forward. Pelvis frontal plane amplitude was not significant $(\mathrm{p}=0,16)$. CONCLUSION: Gait analysis demonstrated differences in the transverse plane amplitude of the pelvis, when comparing the three AFO conditions. The assistance of a propulsion system simulating the action of the triceps sural muscle might be able to increase pelvic rotation in the transverse plane, positively impacting gait pattern. Pelvic rotation may influence other gait parameters such as step length and symmetry between limbs, which should be investigated in the future.
\end{abstract}

Keywords: Kinematics; Locomotion; Nervous System Diseases; Orthotic Device.

\section{Introduction}

Ankle foot orthosis (AFO) plays an important role in treatment of patients with neuromuscular impairments, such as cerebral palsy (CP) [1]. The AFOs are prescribed aiming the prevention and/or correction of foot deformities, function improvement, ankle efficiency and performance, facilitating functional activities, such as gait, of children with CP [2,3]. Therefore, the non-use of AFOs by patients with $\mathrm{CP}$ can deteriorate their gait and joints range of motion [4].

There are several types of AFOs commonly prescribe for CP population [6,7]. Hinged AFO (HAFO) is an example of orthosis which blocks plantar flexion without restricting ankle dorsiflexion and is often used in children with $\mathrm{CP}$ and equine gait pattern - excessive plantar flexion caused by spasticity and/or contracture of plantar flexor muscles [7]. HAFO in these cases (children with excessive plantar flexion gait pattern) is indicated to assist some alterations that might be present during their gait, as compromise stability, due to loss of progression and smaller support base, lower speed gait and the shorter step length [5]. Despite short- and long-term benefits, the continuous use of AFOs causes impairments, such as atrophy of the plantar flexor muscles due to ankle disuse through movement restriction.

Once the ankle during gait, through plantar flexor muscles, is responsible for $2 / 3$ of the energy generated in terminal stance phase [8], the use of AFOs, can affect the gait puff-off phase, restricting movement and atrophying ankle muscles. 
Consequently, an inefficient push-off might generate kinematics compensations distant from the ankle, due to force transmission via body interactions of kinetic chain. An example would be the coupling between the foot and pelvis movements $[9,10]$, which plays an important role in gait temporal parameters.

Considering that children with CP might have their push-off affected by an impaired plantar flexor muscles, an orthosis with external propulsion system can compensate the muscle inefficiency of these individuals. The purpose of an AFO with propulsion system would be to simulate the triceps sural muscle during stance phase of gait, minimizing the impact on terminal stance phase and, thus, possible compensations.

In this context, tridimensional (3D) printed devices allows a more accurate biomechanical analysis of the disadvantages of these devices, through the manufacture of more specific models for each patient, with personalized and functional design. Therefore, improvement of gait pattern is an important goal in treatment of children with $\mathrm{CP}$, due to its impact on autonomy and participation of these children in society [2]. Thus, the present pilot study objective is to verify whether there is a difference between conventional polypropylene AFO and 3D printing AFO, with and without ankle external propulsion system, in pelvis kinematics during the gait of children with $\mathrm{CP}$.

\section{Methods}

\subsection{Sample}

This cross-sectional pilot study was conducted with five children, recruited in Rehabilitation Association of Minas Gerais (AMR). The inclusion criteria were (1) age between 5 and 17 years old; (2) diagnostic of hemiplegic spastic CP; (3) use daily unilateral HAFO; (4) have not undergone botulinum toxin and/or orthopedic surgery on lower limb in the last six months. Children were excluded if they report pain or discomfort during data collection. This study was approved by the Ethics Committee (CAAE: 22988719900005149).

\subsection{Procedures}

The children were instructed to walk at their self-selected walking speed in a 5 meters walkway in three conditions, conventional AFO and 3D printing AFOs with and without external propulsion system, while kinematic data were captured by a tridimensional motion analysis system (11 cameras Oqus 7+, Qualisys Track Motion, Sweden). Conditions order were randomized, and eight to ten minutes rest period were given between each trial. Three force platforms were used to determined heel strike and toe-off events.

\subsection{Data Reduction and Analysis}

Passive markers were placed in plegic limb with orthosis to analyzed kinematic data during gait stance phase. Amplitude pelvis movements in relation to the trunk in frontal and transverse planes were analyzed and compared between conditions.

Repeated measures one-way ANOVA was used to verify whether there is a difference between pelvis amplitude and conventional AFO, 3D printing AFO without external propulsion system, and 3D printing AFO with external propulsion system. All data were processed in SPSS Software (version 21.0) considering $\alpha=0.05$.

\section{Results}

Mean curves angles of pelvis amplitudes in frontal and transverse plane in all three conditions are displaced in Figure 1. Results from ANOVA analysis, and variables descriptive results are shown in Table 1. Only amplitude of the pelvis in transverse plane was different between conditions. 


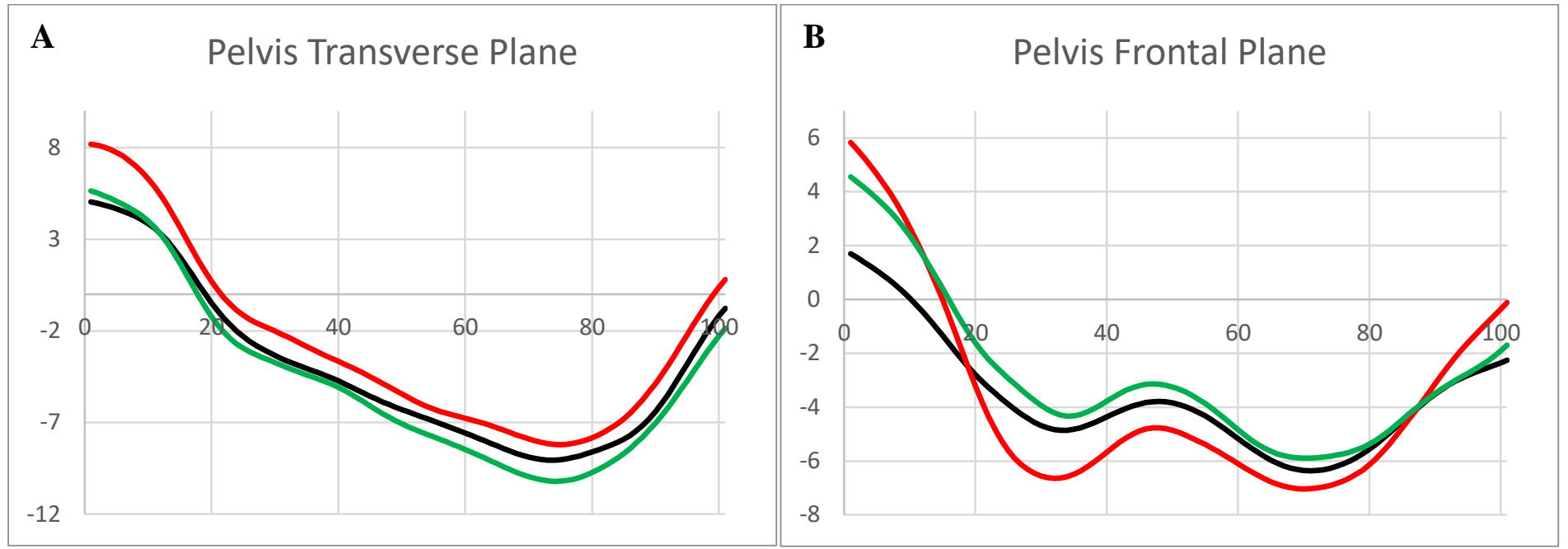

Figure 1: Joint angles $\left({ }^{\circ}\right)$ curves (y-axis) and the percentage of stance phase of gait (\%) (x-axis) of pelvis kinematics. (A) Pelvis transverse plane movements - negative values: ipsilateral rotation, positive values: contralateral rotation; (B) Pelvis frontal plane movements - negative values: abduction, positive values: adduction. Black line - Conventional AFO; Green line - 3D printing AFO without external propulsion system; Red line - 3D printing AFO with external propulsion system.

Table 1. Mean, standard deviation and 95\% Confidence Interval and ANOVA analysis results from amplitudes $\left(^{\circ}\right)$ of the pelvis in transverse and frontal planes during stance phase of gait in the three conditions.

\begin{tabular}{ccc}
\hline & \multicolumn{2}{c}{ Amplitude +- SD (CI) } \\
\hline Conditions & Frontal & Transverse \\
\hline Conventional AFO & $\begin{array}{c}10.5+-4.6^{\circ} \\
(4.76-16.22)\end{array}$ & $\begin{array}{c}15.2+-5.6^{\circ} \\
(8.28-22.23)\end{array}$ \\
& & \\
3D printing AFO & $12.24+-8.02^{\circ}$ & $16.8+-5.08^{\circ}$ \\
without Propulsion & $(2.28-22.2)$ & $(10.48-23.1)$ \\
3D printing AFO & $14.68+-5.85^{\circ}$ & $17.61+-5.41^{\circ}$ \\
with Propulsion & $(7.41-21.94)$ & $(10.88-24.33)$ \\
\hline F (2,8) & 2.243 & 4.538 \\
p (ANOVA) & 0.16 & $\mathbf{0 . 0 4}$ \\
\hline kle Foot Orthosis; SD - Standard Deviation; CI $(95 \%$ Confidence Interval)
\end{tabular}

\section{Discussion}

This pilot study verified the difference between three conditions, conventional polypropylene AFO and 3D printing $\mathrm{AFO}$, with and without ankle external propulsion system, in pelvis kinematics during the gait of children with $\mathrm{CP}$. Transverse and frontal plane pelvis amplitudes of the plegic lower limb were analyzed, but only transverse plane showed statistical differences.

Pelvis movements in transverse plane assists the movement of the body forward [11]. Therefore, it is possible that with more energy reaching the pelvis coming from the ankle through external propulsion system of 3D printing, pelvis movements may also increase, facilitating contralateral lower limb passage to the front. This reasoning is reinforced by the coupling and force transmission that occur between the foot and pelvis [9,10], and might be supported partially by the presented results. 
In addition, pelvis movements plays an important role in regulating step length [11]. Specifically, pelvis rotation in transverse plane increases the forward swing of the contralateral limb and consequently increasing step length of the limb with the orthosis [11]. Since it is common individuals with CP have shorter step length in the plegic limb, increasing the orthosis leg step length, may also increase the symmetry between the limbs.

Despite the statistical analysis used in this pilot study only reveals differences between conditions, without demonstrated the location of this difference, the examination of the descriptive data shows an increase of pelvis amplitude degrees on both, transverse and frontal, planes. Besides that, all AFOs have the same principles, the material and manufacturing process are the only alterations among conventional polypropylene and 3D printing AFOs, being the propulsion system the single modification between the orthoses. Thus, we can infer that the external propulsion system of the 3D printing AFO is responsible for those changes. Furthermore, the amplitude of the pelvis in frontal plane may not have revealed difference between conditions because of the small sample size, since it is a pilot study. Therefore, the study continuation might clarify the assumptions.

\section{Conclusion}

Children tridimensional gait analysis demonstrated differences in the amplitude of the pelvis in transverse plane, after comparisons of two types of AFOs, differing on their manufacturing process, in three conditions (conventional polypropylene and 3D printing AFO, with and without ankle external propulsion system). Therefore, an external propulsion system simulating the triceps sural muscle action might be able to increase pelvic rotation in the transverse plane, positively impacting the children gait pattern. Pelvic rotation may influence other gait parameters such as step length and symmetry between limbs, which should be investigated in future researches.

\section{Acknowledgements}

All financial resources necessary for the project execution were acquired through the Brazilian Ministry of Health through PRONAS/PCD program, numbered as SIPAR:25000163653/2014-33.

\section{References}

[1] J.A. CARVALHO, Órteses: um recurso terapêutico complementar, 1st editio, Manole, Campinas, SP, 2006.

[2] M. Lintanf, J.S. Bourseul, L. Houx, M. Lempereur, S. Brochard, C. Pons, Effect of ankle-foot orthoses on gait, balance and gross motor function in children with cerebral palsy: a systematic review and meta-analysis, Clin. Rehabil. 32 (2018) 1175-1188.

[3] K. Bjornson, C. Zhou, S. Fatone, M. Orendurff, R. Stevenson, S. Rashid, The effect of ankle-foot orthoses on community-based walking in cerebral palsy: A clinical pilot study, Pediatr. Phys. Ther. 28 (2016) 179-186.

[4] A.J. Ries, T.F. Novacheck, M.H. Schwartz, A data driven model for optimal orthosis selection in children with cerebral palsy, Gait Posture. 40 (2014) 539-544.

[5] B. Balaban, E. Yasar, U. Dal, K. Yazicioglu, H. Mohur, T.A. Kalyon, The effect of hinged ankle-foot orthosis on gait and energy expenditure in spastic hemiplegic cerebral palsy, Disabil. Rehabil. 29 (2007) 139-144.

[6] M. Buckon, Cathleen E; Thomas, S S; Jakobson-Huston, S; Moor, M; Sussman, Michael; Aiona, Comparison of three ankle - foot orthosis configurations for children with spastic hemiplegia, Dev. Med. Child Neurol. 43 (2001) 371-378.

[7] J. Rodda, H.K. Graham, Classification of gait patterns in spastic hemiplegia and spastic diplegia: A basis for a management algorithm, Eur. J. Neurol. 8 (2001) 98-108.

[8] K.E. Zelik, P.G. Adamczyk, A unified perspective on ankle push-off in human walking, J. Exp. Biol. 219 (2016) 3676-3683.

[9] K. Duval, T. Lam, D. Sanderson, The mechanical relationship between the rearfoot, pelvis and low-back, Gait Posture. 32 (2010) 637-640.

[10] R.A. Resende, K.J. Deluzio, R.N. Kirkwood, E.A. Hassan, Increased unilateral foot pronation affects lower limbs and pelvic biomechanics during walking, Gait Posture. 41 (2015) 395-401. 
[11] H. Saunders, JB; Inman, VT; Eberhart, The major determinants in normal and pathological gait, J Bone Jt. Surg Am. 35 (1953) 543-558. 